\title{
UAS-supported Digitalized Search-And-Rescue using Harmonic Radar Reflection
}

J onatan Olofsson, Tomas Forssén, Gustaf Hendeby, Isaac Skog and Fredrik Gustafsson

The self-archived postprint version of this journal article is available at Linköping University Institutional Repository (DiVA):

http:// urn.kb.se/ resolve?urn=urn:nbn:se:liu:diva-166539

N.B.: When citing this work, cite the original publication.

Olofsson, J., Forssén, T., Hendeby, G., Skog, I., Gustafsson, F., (2020), UAS-supported Digitalized Search-And-Rescue using Harmonic Radar Reflection, Proceedings of 2020 IEEE Aerospace Conference. https:// doi.org/ 10.1109/AERO47225.2020.9172655

Original publication available at:

https:// doi.org/ 10.1109/AERO47225.2020.9172655

Copyright: Institute of Electrical and Electronics Engineers (IEEE) http://www.ieee.org/index.html @2020 IEEE.

Personal use of this material is permitted. However, permission to reprint/ republish this material for advertising or promotional purposes or for creating new collective works for resale or redistribution to servers or lists, or to reuse any copyrighted component of this work in other works must be obtained from the IEEE. 


\section{UAS-supported Digitalized Search-And-Rescue using Harmonic Radar Reflection}

\author{
Jonatan Olofsson \\ Dept. of Electrical Engineering \\ Linköping University \\ jonatan.olofsson@liu.se
}

\author{
Isaac Skog \\ Dept. of Electrical Engineering \\ Linköping University \\ isaac.skog@liu.se
}

Tomas Forssén

Recco AB

tomas.forssen@recco.com

\author{
Gustaf Hendeby \\ Dept. of Electrical Engineering \\ Linköping University \\ gustaf.hendeby@liu.se
}

\author{
Fredrik Gustafsson \\ Dept. of Electrical Engineering \\ Linköping University \\ fredrik.gustafsson@liu.se
}

\begin{abstract}
Search-And-Rescue (SAR) is one of many fields with applications benefiting from the increasing availability of Unmanned Aerial Systems (UASs). Most UAS applications rely on the UAS's capability to carry a camera and stream video data for manual or automated processing. However, this relies on unobstructed views of the target, which limits the applicability of these systems. In this paper, we instead describe the development and initial application testing of a system with a UAS-carried harmonic radar. This sensor is designed to detect the presence of Recco radar reflectors, commonly found integrated into alpine clothes and gear. The reflectors can be detected through vegetation and snow and is independent of many external factors such as lighting conditions. The paper describes the system design and provides initial real-world results. The initial tests show fruitful results and opens up several avenues of continued research and development.
\end{abstract}

\section{Table of Contents}

1. Introduction.......................... 1

2. System Description ................... 2

3. Harmonic Radar........................ 3

4. Experiments and Results ................ 4

5. Summary and Discussion............... 5

Acknowledgments....................... 6

References ......................... 6

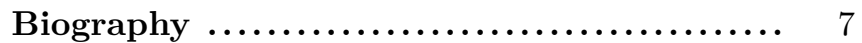

\section{Introduction}

Finding missing people is a time-critical challenge, where the final outcome often depends on the time it takes to find the victim. Only in Sweden, with 10 million people, 7000 persons are reported missing every year, leading to 300 organized Search-And-Rescue (SAR) operations [?]. Organizing a search party takes time, and the search itself is a time-consuming process as well. Manned aircraft and helicopters are frequently used, but generally have long response times and are costly to operate. Consequently, many organizations have started to investigate how Unmanned Aerial Systems (UASs) can be used to facilitate the search. UASs have the advantage of being portable and comparatively cheap, and the rescue personnel can be trained to operate them and start the operation as soon as they reach the area of interest. Field tests have proven that both manned helicopters and UASs with a combination of traditional electro-optical (EO) and thermal infrared (IR) cameras are very promising tools [11] in SAR operations. For example, [7] shows that the time to find a victim can be reduced a factor 10 compared to a traditional SAR operation when using aerial based search tools.

A pre-requisite for this to work is that the operator is trained to both operate the UAS and interpret the video information. Results from the EU project ICARUS indicate that it is this lack of autonomy that is the bottleneck that hinders UAS systems to become a standard tool in SAR operations [5]. A challenge with developing autonomous systems is that IR cameras gives many false positives from wildlife, sun heated stones, etc. Additional complications apply for SAR operations in snow conditions, a common scenario in northern countries. Already a snow layer of $1 \mathrm{dm}$ conceals thermal radiation from a human almost completely, making them invisible in thermal imaging [9]. If a UAS is to be accepted by the operators, then the false alarm rate must be very low and the probability of detection must be high.

The envisioned future approach we foresee for UAS assisted SAR operations is as follows. First, the operator defines a search area and inputs prior information about the regions where the missing person are most likely to be located. Next, geographical information systems are used to infer information based on expert knowledge about how people normally move in different terrain and topography. From this combined information, a nominal search route for the UAS is computed. The UAS then uses radio detectors to look for radio reflectors and transmitters that may belong to the missing person, and trilateration algorithms are used to coarsely locate the source. Once a source is detected and located, a new route is planned to fine tune the position estimate, and cameras can be used to provide additional information about the detected object. This leads to one or more potential victim localizations, from which both EO and IR images are returned to the operator for manual classification and further decision-taking on how to continue the rescue operation. Meanwhile, the UAS can continue its search for more subjects.

The outline of the paper is as follows. In Section 2, the developed system is described as a whole. Section 3 


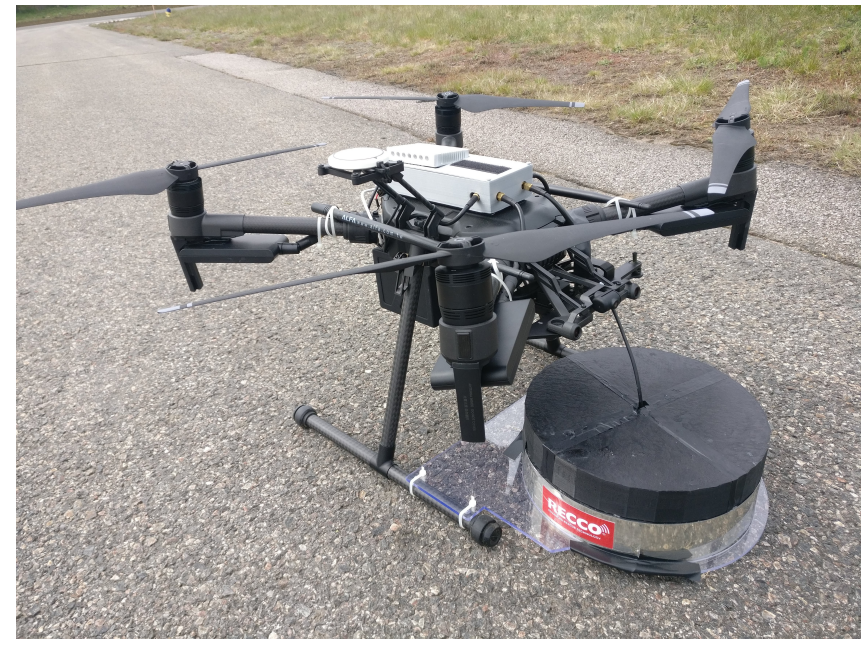

Figure 1: The Recco system - the cylindrical box at the front - was mounted on a DJI Matrice 210 system along with the main onboard computer (gray box) which collects radar measurements and system poses and transmits them to a server for storage.

then continues to go into the details about the specific sensor that this article is focused on, i.e. the Recco harmonic radar for UAS use. Section 4 describes the field tests conducted to validate the initial prototype, and presents the results. Section 5 discusses bottlenecks in the current design, proposes next steps and lessons learnt, and concludes the paper.

\section{System Description}

The UAS based SAR system comprises three major parts - a harmonic radar unit, a UAS carrier, and a backend for data storage and visualization. The carrier in this system is a DJI Matrice 210 UAS onto which the radar unit and an onboard computer were attached. The setup is shown in Figure 1. In this setup the UAS is further equipped with antennas for WiFi and Bluetooth communication and scanning, although those were not active in the experiments described in this article. The gray box on top is the main onboard computer. The Recco radar is seen as the cylindrical box in Figure 1.

A systemic outline of the system is shown in Figure 2, with a summary of its interconnections. Following the general flow of information from the sensor to the user, the functionality of the different parts are as follows.

(1) Recco radar detector This is the main sensor used in this setup. It is further described in Section 3. To provide stable voltage independent of the rest of the system, the transmitter is independently powered from a separate battery. Meanwhile, the detector controller is powered over USB connection, which also serves as the datalink to the system's main onboard computer. Most importantly, the sensor delivers a time-stamped metric of the radar response.

(2) UAS Carrier The carrier, a DJI Matrice 210, has two purposes. Besides physically carrying the system, it also provides in flight pose estimates. It is interfaced

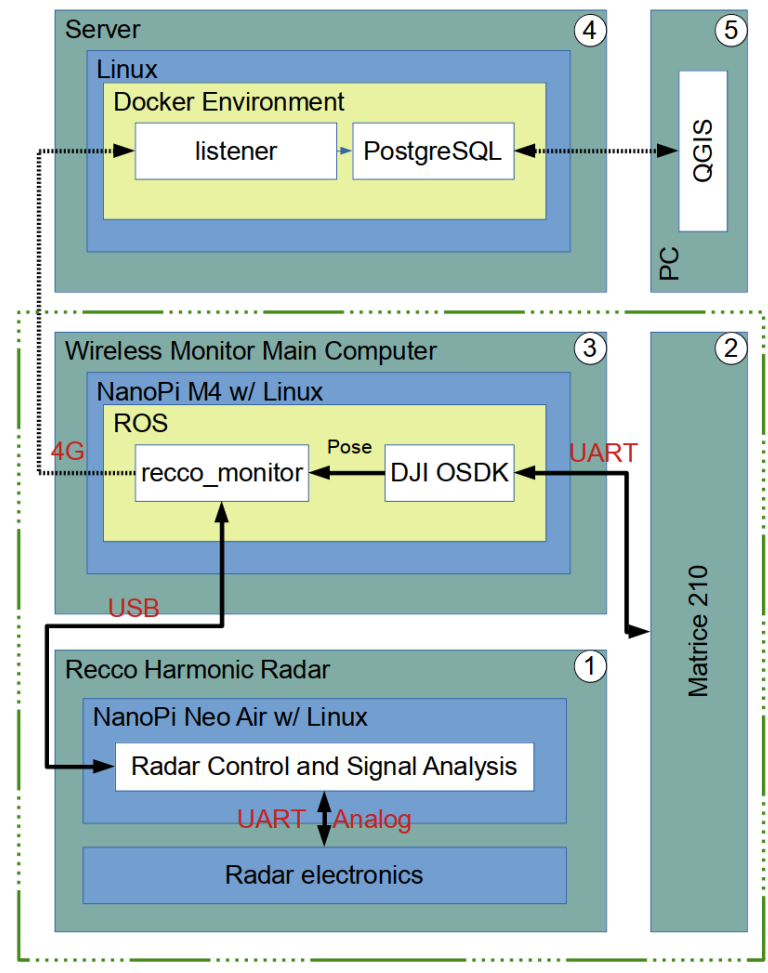

Figure 2: Systemic outline of the full system used for flight, data collection, storage and visualization.

using the DJI Onboard SDK over a UART connection to the main onboard computer. The pose is given at a rate of $10 \mathrm{~Hz}$ - a factor 10 times faster than the radar is sampled - to be able to be match together pose and radar response as close as possible in the main computer. (3) Main Onboard Computer The main onboard computer constitutes the conceptual center of the system, where sensor data from all system components is collected and put together. Most importantly, radar data and pose estimates are put together and then sent over an SSL-encrypted $4 \mathrm{G}$ link with protobuf encoding to the backend server. The implementation uses the Robot Operating System (ROS) framework.

(4) Backend server The backend server is set up to listen for incoming protobuf data, storing all incoming measurements in a PostgreSQL/postgis database. The listener is implemented as a python executable, running in a Docker environment.

(5) Visualization PC By using the postgis storage format, the database on the backend can be visualized in real time using e.g., — as shown in Figure 3 - QGIS. 


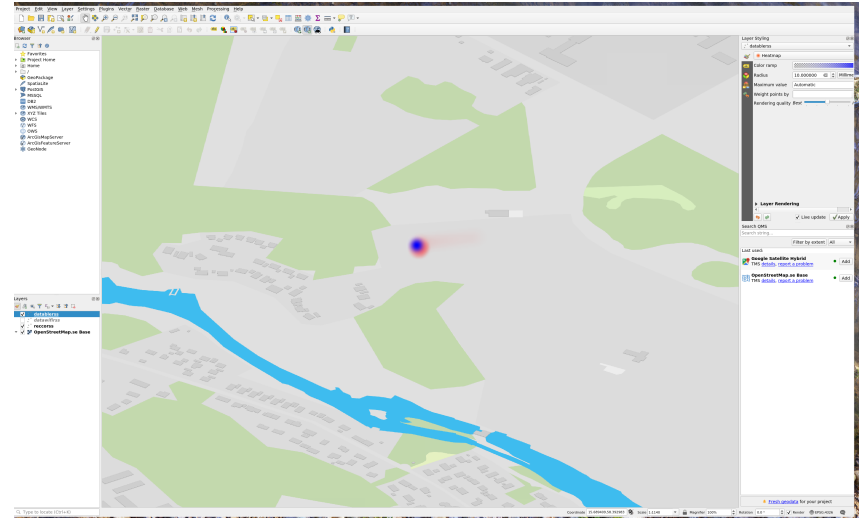

Figure 3: By using the postgis storage format, the postgis database on the backend can be visualized in real time using QGIS.

\section{Harmonic Radar}

A harmonic radar system, such as developed by Recco, comprises two parts - a detector and a reflector. The detector emits a directional radar signal. When the radar signal hits the reflector, the signal is transformed and reflected back to the detector, thus indicating the existence and direction of a potential victim to the rescuer. The Recco reflector is a passive unit with no need for a built in power supply. The reflectors are currently integrated in products such as jackets, pants, helmets, backpacks, back protectors, boots, detectors, watches, and harnesses. Reflectors are also available as a stand-alone product to be attached to, e.g., helmets and backpacks.

\section{Harmonic Radar Reflections}

The principle of harmonic radar starts with the transmission of an electromagnetic (EM) signal of a specified frequency. When this signal reaches an antenna, its electrons will interact with the signal and induce currents which in turn leads to the phase-shifted reflection/retransmission of the incoming signal. If the antenna is modified with a nonlinear circuit, additional frequencies will be transmitted from the antenna. This radar response is not generally found naturally, which makes it a distinct signal to look for in a natural environment, yielding few false positives.

For a transmitted signal with base frequency $f_{0}$, the main reflected frequency that the detector listens for is the second harmonic of the base frequency, i.e., $2 f_{0}$. This high-level concept of harmonoc reflection is illustrated in Figure 4.

The nonlinear circuit in the reflector can be constructed using simple electronic components [2,6], even using just a single diode [2] and a wire antenna, such as outlined in Figure 5 [3]. This also means that other electronic equipment, such as mobile phones, cameras, etc. may be located using a Recco system.

In Figure 6, an example of what a nonlinear circuit can do to the frequency content is given for an idealized circuit. This shows that the ideal halfwave rectification of an incoming signal generates a signal with, among others, twice the frequency of the incoming signal, for the

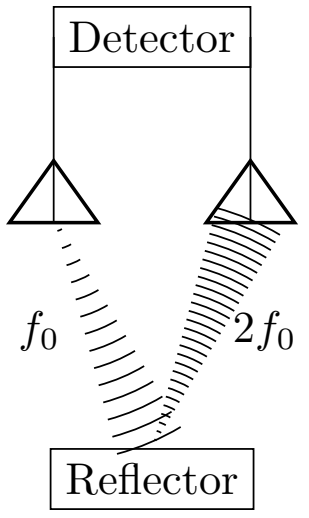

Figure 4: In a harmonic radar setup, a base frequency $f_{0}$ is transmitted, and reflections of a nonlinear reflector is listened for at twice the base frequency.

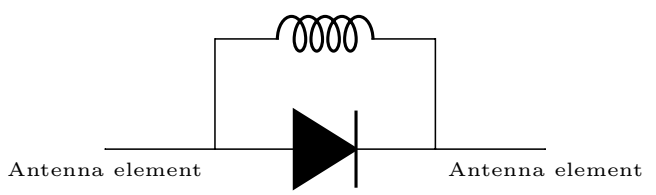

Figure 5: Example of a simple harmonic reflector circuit.

antenna to emit. This broadly motivates listening for the double frequency as a means to detect the presence and direction of a reflector matched with the base frequency.
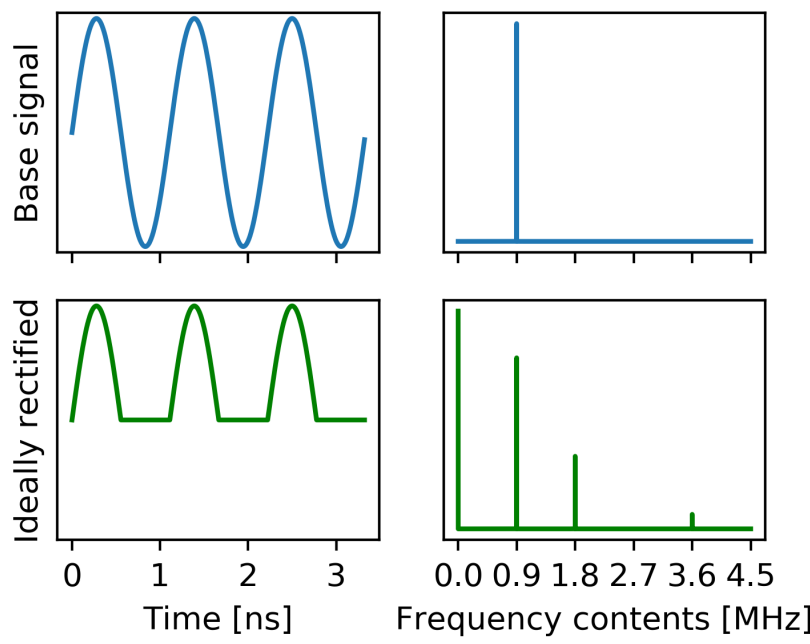

Figure 6: The frequency contents of an ideally half wave rectified sinusoid signal.

Today, harmonic radar has two main civilian applications in the literature. One is the low-energy, portable, SAR application for uniformed blue-light forces that is discussed in this paper, as well as, e.g., by [6]. The other is less-portable high-energy, high-frequency tracking of, e.g., bees and other insects $[1,4,8,10]^{2}$.

${ }^{2}$ Including references 


\section{UAS-Mounted Harmonic Radar}

The currently available Recco systems are operated by humans. If a reflector is within range and Field-of-View (FOV) of the detector, an acoustic signal is given to the operator. The signal strength of the acoustic signal is directly related to the strength of the signal received back from the reflector. The strength is maximized when the detector points directly at the reflector, and is further increased if the distance to the reflector is decreased until the detector saturates. The detector has adjustable output power, and by lowering the output power the direction to a reflector can be pin-pointed with high accuracy at close range. The handheld Recco system can locate Recco reflectors at a range of up to $80 \mathrm{~m}$ in air and $20 \mathrm{~m}$ through packed snow. Recco has also recently deployed a Recco SAR system using a detector designed to be carried by a helicopter. Searching from a height of $100 \mathrm{~m}$ will cover a search area that is about $100 \mathrm{~m}$ wide, enabling the helicopter to search an area of $1 \mathrm{~km}^{2}$ within 6 min.

The Recco detector in this project is developed to be carried by a professional grade UAS. This restricts the detector in both size and weight. The detector used in this project is a cylinder $25 \mathrm{~cm}$ in diameter and $10 \mathrm{~cm}$ in height. The weight is about $800 \mathrm{~g}$. The diameter is mostly restricted by the operational frequency of the Recco system, which is in the $900 \mathrm{MHz}$ band. Unlike the handheld device, the output power is controlled remotely through a USB interface and, instead of a human ear, an algorithm processes the digitalized audio signal and calculates the amplitude of the detection signal. The output power can be controlled in eight discrete settings, $1-8$.

The DJI Matrice 210 has an ultrasonic sensor mounted facing down from the main body, which limits the UAS's capability of carrying cargo directly below the body. This currently entails a slightly awkward offcenter position for the radar. While the UAS is capable of compensating for the weight offset, it has a negative effect on the balance of the UAS and, in particular, the UAS's capability to control its pitch angle due to power limitations on the forward motors. In the future, these problems could be alleviated by circumventing the ultrasonic sensor, or otherwise redistributing the weight of the UAS.

A schematic view of the radar prototype mounting is seen in Figure 7. This also relates the altitude of the UAS with the theoretical area of the ground plane circle of an isometric return signal, given that $a=\pi r^{2}$ and $r \propto \frac{h}{\tan (\alpha)}$. The radius for a given height yields $\alpha$, the effective opening angle of the radar. The area covered by a single detection is in turn proportional to the area covered in a given time, and hence inversely proportional to the expected search time for a given area. It is clearly desirable to increase the detection range to decrease search time. However, since the detectability of the reflectors decreases with range, there is also a maximum area which can be within the FOV with reflectors still being reliably detected.

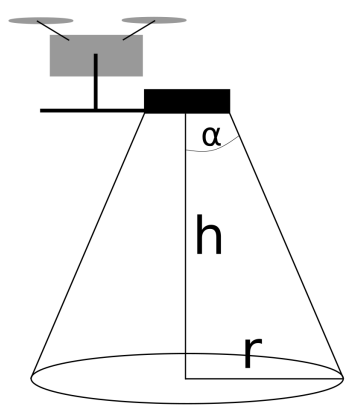

Figure 7: Systemic drawing of the UAS and how its environmental parameters relate. In this paper, the attitude of the UAS is assumed to be such that the Recco detector antenna point straight down.

\section{Experiments and Results}

Three experiments were designed for the initial evaluation of the system. In this paper, the UAS and its detector is considered horizontal. This is a realistic assumption given the low airspeed in the experiments - a faster air speed would require compensation for the angles of the drone and the detector.

\section{Experiment 1: Establish ego disturbance}

The operating range of a Recco system is mainly limited by the output power of the radio signal emitted by the detector. Even though the antenna is constructed such that the power emitted is directed in a narrow lobe towards the ground, a small fraction of power is also emitted towards the UAS. The metals in the UAS, especially the radio systems, will act as reflectors for this back lobe and generate a response back to the detector, which will then detect a false target.

To determine the level of disturbance from the own system, the carrier UAS was positioned such that the radar was out of range of external disturbances - over an isolated forest area at an altitude of $120 \mathrm{~m}$. The radar's transmitted power was then remotely varied and the response was recorded along with signal metrics. In previous tests, the stand-alone radar has been tested successfully on all power levels, making disturbances found in this experiment likely to originate from reflections from the UAS.

As seen in Figure 8, the response from the own system exhibits a very clear knee between output power level 4 and 5. For larger output power levels, the signal saturates. Having the knee at this unexpectedly low output power level shows that additional shielding and filtering is necessary in the next prototype. The exact source of the disturbances is yet to be identified, although several lines of inquiry have been proposed. For instance, the unused radio equipment on the test UAS could be acting as reflectors, in which case those could easily be removed for future flights. Nevertheless, the radar provides true detections reliably at output power level 4 , albeit so far at less range than initially intended. 


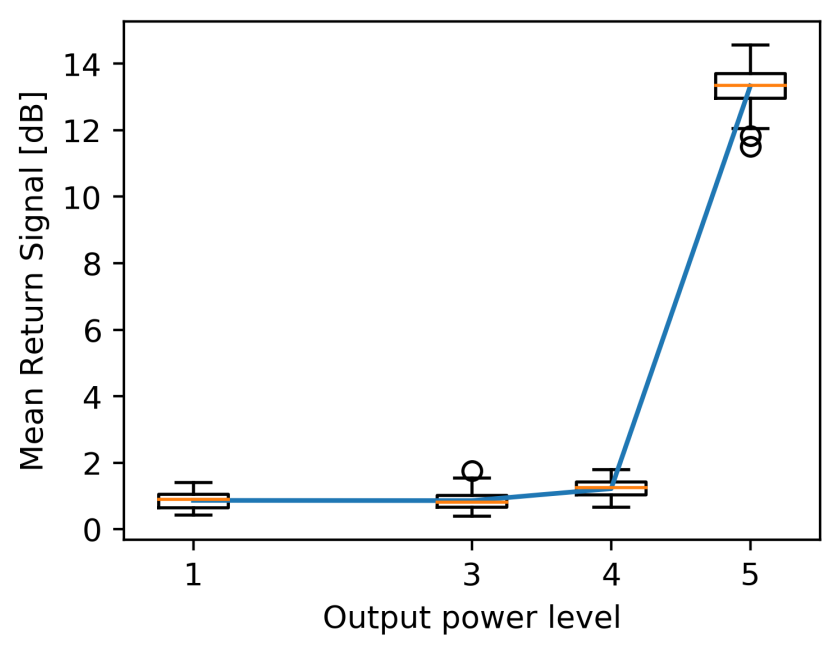

Figure 8: The return from the system itself was tested in order to determine a maximum output power for reliable detection. The blue line shows the mean, while the boxplot is used to indicate the distribution of measurements at each output power level.

\section{Experiment 2: Altitude profiling}

For this experiment, a reflector was placed $40 \mathrm{~cm}$ above the ground. Directly above, with a fixed output power level of 4 , the carrier UAS was positioned at an altitude where a return signal could not be visibly detected in a live plot. The UAS was then slowly lowered while signal metrics were recorded at $1 \mathrm{~Hz}$. The results are presented in Figure 9.

The detector prototype is, at this output level, able to reliably detect the reflector from a height of about $20 \mathrm{~m}$ and below. For a SAR system this needs to be increased with at least a factor of four. Not only to be able to fly higher but also to compensate for reflectors that are partially hidden by, e.g., a human body.

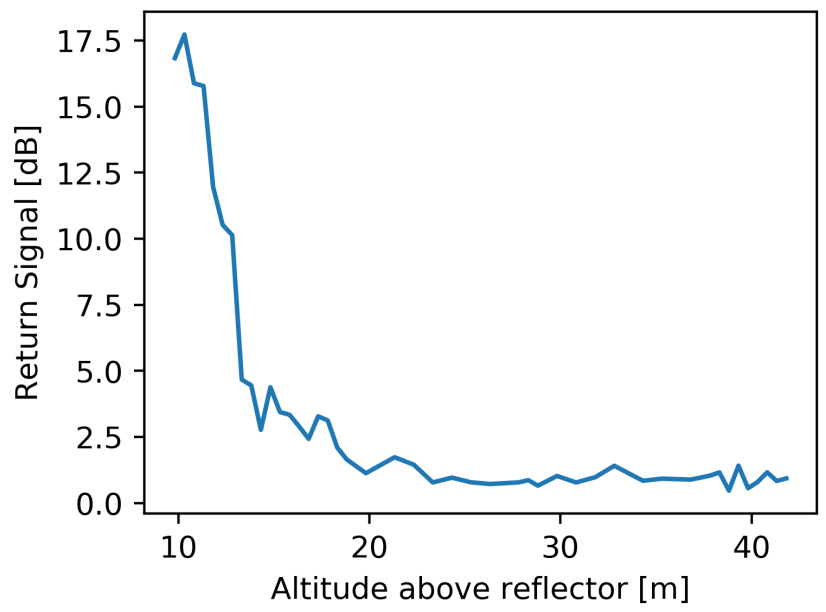

Figure 9: The detector is, on output power level 4 , able to reliably detect a reflector from about $20 \mathrm{~m}$.

\section{Experiment 3: Fixed altitude response mapping}

To profile the radar response over a larger area, the UAS was flown at a fixed altitude in a random pattern over and around the same reflector as in experiment 2 . The heading was kept approximately constant throughout the flight. The radar response was recorded at $1 \mathrm{~Hz}$ along with the UAS position.

With radar output power level 4 , the UAS was kept at an approximately constant altitude of $8 \mathrm{~m}$ over the reflector. The mean return signal strength of a $0.9 \times 0.7 \mathrm{~m}$ grid, smoothed by a $5 \times 5$ Gaussian kernel, is plotted in Figure 10. As seen in the plot, the radar exhibits a ground plane radius of detectability of about $8 \mathrm{~m}$. This corresponds to a FOV angle $\alpha=45^{\circ}$ for an altitude of $8 \mathrm{~m}$. This could be used to infer, e.g., the distance between turns in a search pattern, such as a lawnmower pattern. As the sensor saturates at about $20 \mathrm{~dB}$, it is clear that the target could also have been detectable from a greater altitude, which would further expand the radius of detectability. In a real scenario, one also needs to consider reflectors that are partially hidden. In order to reliably detect such reflectors a stronger output power and/or a lower search altitude is needed.

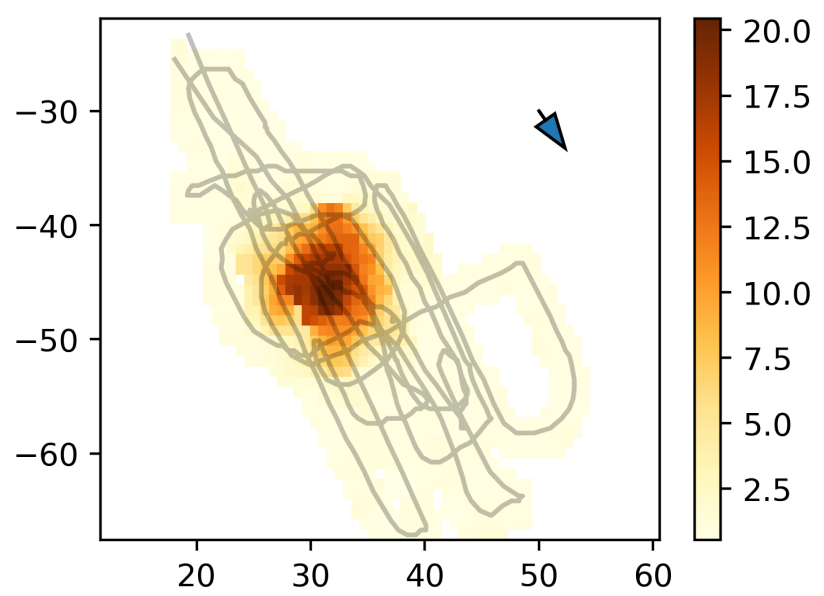

Figure 10: At a fixed height of $8 \mathrm{~m}$ above the reflector, the detectability was mapped by plotting the mean return signal within a mesh grid. The heading of the UAS was kept approximately constant and is indicated by the blue arrow. The flight path is shown in gray.

\section{Summary and Discussion}

In this paper we have presented the design and preliminary prototype evaluation of a sensor for digitalized SAR using harmonic radar reflections. The sensor is mounted on a UAS and integrated into a framework for sensor sampling, pose- and time-stamping, storage and visualization.

In general, most applications of UASs utilize EO or IR cameras to collect and act upon data. In contrast, the system described in this paper provides a sensor previously unavailable for UAS applications. The project has successfully, via real-world experiments, demonstrated the possibility of using UAS-carried harmonic radar for target detection and localization. That said, work still 
remains to decrease the disturbance from the UAS and thereby increase the range.

A number of ideas have been proposed to reduce the disturbance from the UAS. For instance, the system currently is equipped with other antennas to find missing people using, e.g., WiFi and Bluetooth signals. These antennas could be removed for a single-sensor search with the Recco system. Moreover, the position of the detector could be adjusted - possibly with the addition of longer UAS "legs" for a more advantageous disturbance environment, farther away from other electronics. Since the disturbance depends on the output power, the source of disturbance is the radar signal itself. By enhancing the antenna to direct the transmit power in a more narrow beam would also reduce the disturbance from the UAS. The challenge is to keep the size, weight, and formfactor of the antenna within the bounds of what could be carried by a UAS.

With its weight and size, the system requires a fairly large drone to act as carrier although the concept is easily carried over to other manufacturers and models of UASs. The integration with the DJI UAS is lightweight and abstracted and could easily be replaced for other interfaces. While the mounting position on the DJI Matrice 210 allowed the UAS's altitude sensing ultrasonic sensor to work, the imbalance of the drone put undesired stress on the front motors. Moving the sensor or otherwise improving the weight distribution is desirable for a safer workload for a wider range of drones.

In this paper, the UAS and its detector was considered horizontal due to an intentionally low airspeed. To enable proper signal processing at higher airspeeds, the processing would need to include considerations for the full pose of the sensor.

Apart from the previously discussed improvements on disturbance levels, future work includes further verification and data collection. Moreover, the sensor data and heatmaps such as in Figure 10 can be utilized to automatically plan and optimize flight paths based on both other datasources and real-time signals from the Recco system. Through the existing integration with ROS and the UAS control system, the necessary interfaces for this are easily available for future practical implementations and tests, as well as research.

To summarize, the developed prototype has been shown to successfully aid in the localization of a target in the field. Tests and verification flights have been performed and practical aspects have been evaluated. The so far fruitful results show potential for future integration into real world SAR scenarios.

\section{Acknowledgments}

This project has been performed with support from Sweden's Innovation Agency, project nr. 2018-01762, "Digitalized Search and Rescue".

\section{References}

[1] H. Aumann, E. Kus, B. Cline, and N. W. Emanetoglu, "A low-cost harmonic radar for tracking very small tagged amphibians," in 2013 IEEE International Instrumentation and Measurement Technology Conference (I2MTC). IEEE, may 2013. [Online]. Available: https: //doi.org/10.1109/i2mtc.2013.6555415

[2] G. Charvat, E. Rothwell, and L. Kempel, "Harmonic radar tag measurement and characterization," in IEEE Antennas and Propagation Society International Symposium. Digest. Held in conjunction with: USNC/CNC/URSI North American Radio Sci. Meeting (Cat. No.03CH37450). IEEE. [Online]. Available: https://doi.org/10.1109/aps.2003.1219331

[3] D. Cohen, "The design of a harmonic radar system," 2018.

[4] B. Colpitts and G. Boiteau, "Harmonic radar transceiver design: Miniature tags for insect tracking," IEEE Transactions on Antennas and Propagation, vol. 52, no. 11, pp. 2825-2832, nov 2004. [Online]. Available: https://doi.org/10.1109/ tap.2004.835166

[5] G. D. Cubber, D. Doroftei, K. Rudin, K. Berns, A. Matos, D. Serrano, J. Sanchez, S. Govindaraj, J. Bedkowski, R. Roda, E. Silva, and S. Ourevitch, "Introduction to the use of robotic tools for search and rescue," in Search and Rescue Robotics. Rijeka: IntechOpen, 2017, ch. 1. [Online]. Available: https://doi.org/10.5772/intechopen.69489

[6] P. Fuks, Harmonic Radar, a Modern Method for Location of Avalanche Victims, ser. TRITA / TET. Division of Electromagnet. Theory, the Royal Inst. of Technology, 1981.

[7] Y. Karaca, M. Cicek, O. Tatli, A. Sahin, S. Pasli, M. F. Beser, and S. Turedi, "The potential use of unmanned aircraft systems (drones) in mountain search and rescue operations," The American Journal of Emergency Medicine, vol. 36, no. 4, pp. 583-588, Apr. 2018. [Online]. Available: https://doi.org/10.1016/j.ajem.2017.09.025

[8] M. O'Neal, D. Landis, E. Rothwell, L. Kempel, and D. Reinhard, "Tracking insects with harmonic radar: a case study," American Entomologist, vol. 50, pp. 212-218, 122004.

[9] M. Silvagni, A. Tonoli, E. Zenerino, and M. Chiaberge, "Multipurpose uav for search and rescue operations in mountain avalanche events," Geomatics, Natural Hazards and Risk, pp. 1-16, 102017.

[10] N. Tahir and G. Brooker, "Toward the development of millimeter wave harmonic sensors for tracking small insects," IEEE Sensors Journal, vol. 15, no. 10, pp. 5669-5676, oct 2015. [Online]. Available: https://doi.org/10.1109/jsen.2015.2445933

[11] C. V. Tilburg, "First report of using portable unmanned aircraft systems (drones) for search and rescue," Wilderness 83 Environmental Medicine, vol. 28, no. 2, pp. 116-118, Jun. 2017. [Online]. Available: https://doi.org/10.1016/j.wem.2016.12. 010 


\section{Biography}

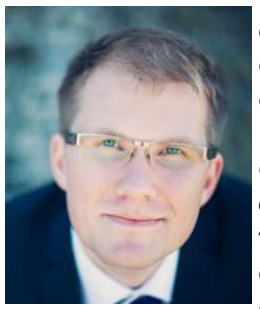

Jonatan Olofsson is a postdoctoral researcher in the Division of Automatic Control, Department of Electrical Engineering, Linköping University, Sweden. Research interests include the field of sensor fusion, and its practical applications to realworld data. He received his M.Sc. degree in Applied Physics and Electrical Engineering from Linköping University, Sweden, in 2012 and 2019 his Ph.D. degree in the field of Cybernetics at the Norwegian University of Science and Technology (NTNU) through MarineUAS, a Marie Skłodowska-Curie ITN performing research on the use of Unmanned Aerial Systems on the European coast.

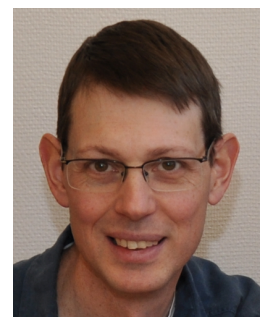

Tomas Forssén is currently working as a Radio Specialist at Recco $A B$. His work involves design of antennas, electronic- and software platforms for harmonic radars and reflectors. Related work also involves development of computational tools for electromagnetic problems and non-linear systems. He received his $M S c$ degree in Electrical Engineering from the KTH Royal Institute of Technology, Stockholm, Sweden in 1999. He received his Ph.D. degree in Signal Processing with a thesis on Parameter Estimation and Waveform Fitting for Narrowband Signals. Between 2005 and 2011 he worked at the GSM Base Station Software group at Ericsson AB. The work included algorithm development for estimation, detection, and demodulation problems. He also participated as a standardization delegate in $3 G P P$ where he was responsible for radio transmitter and receiver performance, and for specifying the physical related layers in GSM.

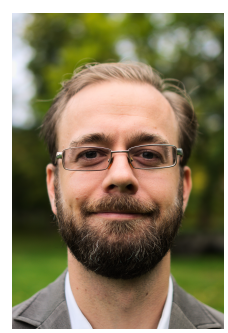

Gustaf Hendeby is Associate Professor in the Division of Automatic Control, Department of Electrical Engineering, Linköping University. He received his $M S c$ in Applied Physics and Electrical Engineering in 2002 and his PhD in Automatic Control in 2008, both from Linköping University. He worked as Senior Researcher at the German Research Center for Artificial Intelligence (DFKI) 20092011, and Senior Scientist at Swedish Defense Research Agency (FOI) and held an adjunct Associate Professor position at Linköping University 2011-2015. Dr. Hendeby's main research interests are stochastic signal processing and sensor fusion with applications to nonlinear problems, target tracking, and simultaneos localization and mapping (SLAM). He has experience of both theoretical analysis as well as implementation aspects.

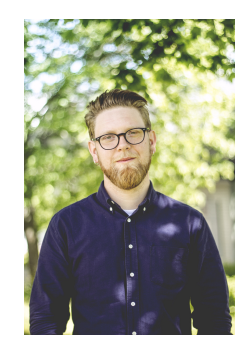

Isaac Skog (S'09-M'10) received his BSc and MSc degrees in Electrical Engineering from the KTH Royal Institute of Technology, Stockholm, Sweden in 2003 and 2005, respectively. In 2010, he received a Ph.D. degree in Signal Processing with a thesis on low-cost navigation systems. In 2009, he spent 5 months with the Mobile Multi-Sensor System research team, University of Calgary, Canada, as a visiting scholar and in 2011 he spent 4 months at the Indian Institute of Science (IISc), Bangalore, India, as a visiting scholar. Between 2010 and 2017 he was a researcher at KTH Royal Institute of Technology, Stockholm, Sweden. During the spring of 2019 he was a visiting researcher at the underwater research department at the FOI Swedish Defence Research Agency. Currently he is an assistant professor at Linköping University, Linköping, Sweden.

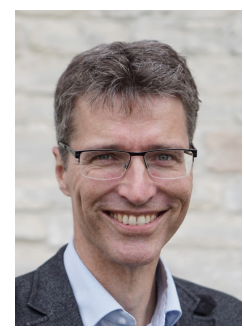

Fredrik Gustafsson is professor in Sensor Informatics at Department of Electrical Engineering, Linköping University, since 2005. He received the M.Sc. degree in electrical engineering 1988 and the Ph.D. degree in Automatic Control, 1992, both from Linkoping University. His research interests are in stochastic signal processing, adaptive filtering and change detection, with applications to communication, vehicular, airborne, and audio systems. He is a co-founder of the companies NIRA Dynamics (automotive safety systems), Softube (audio effects) and Senion (indoor positioning systems).

He was an associate editor for IEEE Transactions of Signal Processing 2000-2006, IEEE Transactions on Aerospace and Electronic Systems 2010-2012, and EURASIP Journal on Applied Signal Processing 20072012. He was awarded the Arnberg prize by the Royal Swedish Academy of Science (KVA) 2004, elected member of the Royal Academy of Engineering Sciences (IVA) 2007, and elevated to IEEE Fellow 2011. He was awarded the Harry Rowe Mimno Award 2011 for the tutorial "Particle Filter Theory and Practice with Positioning Applications", which was published in the AESS Magazine in July 2010, and was co-author of "Smoothed state estimates under abrupt changes using sum-ofnorms regularization" that received the Automatica paper prize in 2014. 